\title{
Molisch 反应：发现、原理及应用
}

郝志友 ${ }^{*}$, 陈辉, 曹彦刚, 孙彦君, 李红伟, 郑晓珂, 冯卫生 ${ }^{*}$

河南中医药大学药学院, 郑州 450046

摘要: Molisch反应发现于1886年, 此后对糖和苷类化合物的研究提供了强大的定性鉴别工具。本文回顾了Molisch 反应的发现过程、反应机理研究及应用, 并对该反应在未来的应用进行了展望。

关键词: Molisch反应; 反应原理; 应用

中图分类号: G64; O6

\section{Molisch's Reaction: Discovery, Mechanism and Application}

\author{
Zhiyou Hao *, Hui Chen, Yangang Cao, Yanjun Sun, Hongwei Li, Xiaoke Zheng, Weisheng Feng * \\ School of Pharmacy, Henan University of Chinese Medicine, Zhengzhou 450046, China.
}

\begin{abstract}
After the discovery in the year 1886, Molisch's reaction used to be applied as a powerful tool to differentiate carbohydrates and glycosides qualitatively from other materials. A brief review is given herein on the discovery, reaction mechanism and application of Molisch's reaction. Perspectives are also given on the potential applications of Molisch's reaction.
\end{abstract}

Key Words: Molisch's reaction; Reaction mechanism; Application

Molisch反应由Hans Molisch发现, 又叫 $\alpha$-芸酚-浓硫酸反应、莫立许反应等, 是一个重要的鉴别 糖和苷类化合物的化学反应。由于其反应简便、灵敏的优点, 在糖和苷类研究历史中起到了极大的 作用。然而, 也许是圊于篇幅限制, 在生物化学、药物化学、中药化学、天然药物化学、食品化学 等相关专业课程的教材中, 只是对Molisch反应原理做粗略介绍, 甚至不做介绍, 更遑论其发现过程 及其拓展应用。本文根据原始文献, 对Hans Molisch的学术经历, Molisch反应的发现、原理及应用 进行回顾, 供相关教师在课堂上参考应用, 以提高相关专业大学生对该知识点的学习兴趣, 拓展其 知识范围, 并期望今后该反应在糖的定量研究上起到更大的作用。

\section{Hans Molisch简介}

汉斯·莫立许(Hans Molisch, 1856-1937) ${ }^{[1,2]}$ 生于捷克布隆(Brünn, 今捷克布尔诺), 是一位苗圃主 人的儿子, 儿时帮助他的父亲进行苗戋种植工作, 为他成为一个著名的植物生理学家培养了浓厚的 兴趣。1897年, 他开启了他的热带植物学之旅, 并在印度尼西亚茂物进行了一段时间的植物生理学 研究, 后经由中国、日本、美国返回捷克布拉格。1909年, 被任命为维也纳大学植物生理学系主任, 直到1928年, 退休后他被任命为名誉教授。在此期间, 他接到邀请, 担任日本东北大学新成立的生

收稿: 2021-01-12; 录用: 2021-03-03; 网络发表: 2021-03-25

“通讯作者, Emails: hzy@hactcm.edu.cn (郝志友); fwsh@hactcm.edu.cn (冯卫生)

基金资助：国家重点研发计划 “中医药现代化研究” 重点专项(2017YFC1702801); 河南省科技攻关项目(212102311091) 
物研究所的负责人三年, 随后他到加尔各答玻色研究所(Bose Institute)讲课和工作, 这些均广泛地扩 展了他的研究领域。

Molisch研究领域非常广泛, 除在植物生理学研究领域著述颇丰外, 还在植物生物学、生物化学、 植物微化学等方面颇有建树。他分离得到许多种类的紫色光合细菌, 包括荚膜红细菌 (Rhodobacter capsulatus), 促进了他细菌光合作用的研究发现。他对植物学和化学的出色研究使他能够将两门学科 结合, 而提出了植物化感作用(allelopathy)的概念, 他是植物化感作用研究的开拓者, 现在已发展成 为独立学科。

\section{Molisch反应的发现}

人们很早就认识到, 糖类物质深度参与了植物的生理、生化过程, 是一类及其重要的化合物。 因此, 就需要研究者用较敏锐的方法检识微量糖类物质是否存在。在Molisch的时代, 糖类物质的鉴 定反应包括Trommer反应 ${ }^{[3]}$ 、Fehling 反应 ${ }^{[3]}$ 等。然而, 这些反应的灵敏度不高、专属性不强, 因此鉴 定糖类化合物的准确度差。

1886年, Molisch在对植物生理学的研究过程中, 偶然发现了一种非常灵敏的检测糖或者苷是否 存在的反应 ${ }^{[4]}$ 。在试管中, 将约 $0.5-1 \mathrm{~mL}$ 的待测溶液滴入两滴 $15 \%-20 \%$ 的 $\alpha$-䒺酚的乙醇溶液, 摇匀, 随后倒入两倍体积的浓硫酸并迅速振摇, 此时如果待测溶液中有糖的存在, 则溶液迅速呈现紫色。 随后用水稀释可使紫色物质沉淀, 该沉淀物可溶于氢氧化钾溶液、乙醇、乙醚和氨水中。Molisch发 现该反应不针对特定类型的糖, 蔗糖、乳糖、葡萄糖、果糖和麦芽糖均发生了显色反应。多元醇类 物质，包括肌醇、甘露醇、卫矛醇和栎醇则不发生显色反应。

Molisch发现, 不仅对糖类化合物可以发生上述反应, 对于糖苷类化合物, 以上反应也可以立即 或在很短的时间内发生。因此, 如果在上述条件下用任何液体获得了紫色物质, 则应推断以上待测 溶液中含糖，或糖苷类化合物在浓硫酸作用下反应生成了糖。

Molisch测试了许多其他类型的有机物, 包括酒精、有机酸、脂肪、苯烃、酚类、树脂、生物碱、 蛋白质等, 均对此反应呈阴性。因此该方法对检出糖和苷的存在具有很好的排他性。

该反应灵敏度极高, 无需加热即可轻松检测到 $0.00001 \%$ 的糖, 灵敏度远超Trommer和Fehling试验。

此外, 当时的人们已经认识到, 糖(葡萄糖)是健康人尿液的组成成分, 糖尿病人尿液中糖的含量 远超正常状态, 而其时检测尿液中糖含量是否偏离正常状态的检测方法不够灵敏。Molisch天才的想 法解决了该问题。其将正常尿液和待测尿液用水稀释至其体积的 100 倍, 均用上述方法反应, 如果待 测尿液反应后出现的紫色要比正常尿液深的多, 则视其为糖尿病尿。或者, 将正常尿液和待测尿液 稀释至其体积的 400-600倍, 如果是糖尿病人的尿液, 即使稀释到如此大的程度, 仍能明显地产生以 上反应现象, 而正常尿液则无明显显色。以上半定量的方法为当时人们诊断糖尿病提供了很好的工 具。

从此以后, 该反应被广为人知, 在植物、动物、真菌中糖或苷类化合物的定性鉴别中被广泛应 用 ${ }^{[5]}$, 并根据它的发明者命名, 被称作莫立许反应(Molisch's Reaction, Molisch's Test)。随着该反应 的广泛应用, 人们对该反应过程进行了改进, 现在一般反应方法如下: 待测溶液约 $1-2 \mathrm{~mL}$ 加入试管, 滴入 $2-3$ 滴 $5 \%$ 的 $\alpha$-菜酚乙醇溶液, 摇勺, 沿试管壁缓慢加入 $1-2 \mathrm{~mL}$ 浓硫酸, 勿振摇。因浓硫酸较重, 因此沉入试管底, 上层为待测液。如果待测溶液中含有糖或苷类化合物, 则在浓硫酸层和待测液层 的交界处，会产生一个紫色的环，因此该反应也称 “紫环反应” (反应流程如图1)。

\section{Molisch反应的原理}

Bredereck研究了Molisch反应的原理 ${ }^{[6,7]}$, 认为单糖首先在浓硫酸作用下脱水缩合生成5-羟甲基糠 醛(由己糖生成)或糠醛(由戊糖生成), 又在浓硫酸作用下, 和两分子 $\alpha$-菜酚缩合生成了化合物 $\mathbf{1}$, 化合 物1迅速在浓硫酸作用下，氧化生成化合物 2 和 3 而显色。反应过程如图2。 


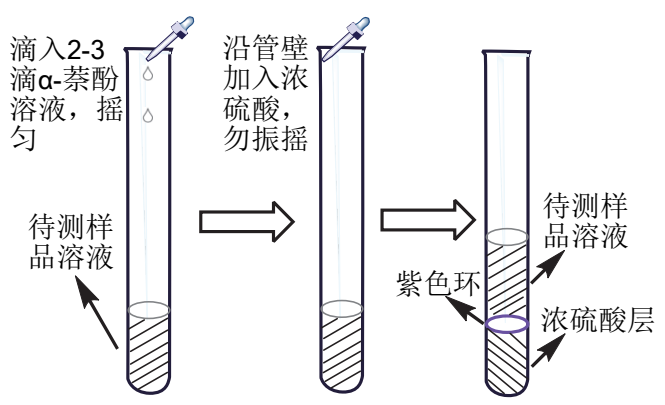

图1 Molisch反应操作流程示意图

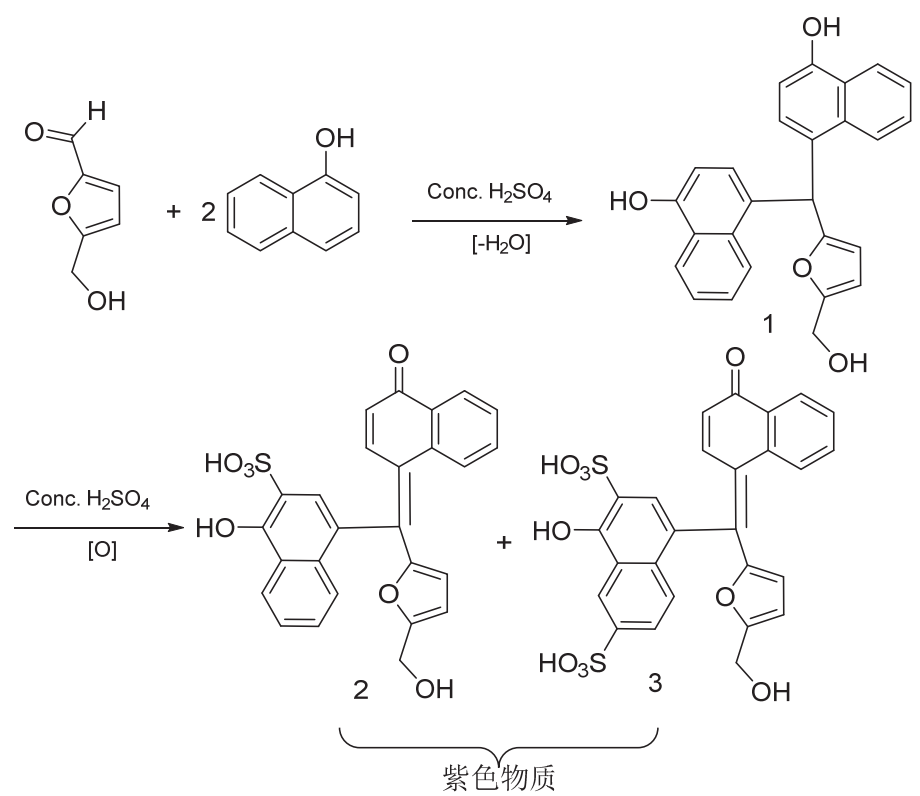

图2 Bredereck的Molisch反应原理

但Mutsuko Ohta等 ${ }^{[8]}$ 对以上推测的结果表示怀疑, 他们认为没有直接的证据表明这些结构的存 在。由于磺化产物的形成, 对Molisch反应产物在结晶状态分离几乎不可能。在浓硫酸存在下, $\alpha$-荎 酚极易在C-2位(然后在C-4位)磺化。磺化反应使反应产物从反应体系中分离, 或者反应产物的结晶, 都变得困难。因此使用衍生化物反应会改善这种情况。Mutsuko Ohta等发现, 用2-甲基- $\alpha$-䒺酚代替 $\alpha$-荎酚和己糖、戊糖反应一样灵敏, 且其有色反应产物易于结晶, 因此可用以推测Molisch反应的机 理。其用葡萄糖或5-羊圣甲基糠醛和2-甲基- $\alpha$-䒜酚、浓硫酸反应后, 反应体系用乙酸乙酯萃取, 萃取 物用硅胶柱色谱分离, 均得到化合物 4 ; 用鼠李糖或 5 -甲基糠醛与2-甲基- $\alpha$-菜酚、浓硫酸反应后, 反 应体系用乙酸乙酯萃取后, 用硅胶柱色谱分离, 均得到化合物 $\mathbf{5}$ 。将 4 、5 的紫外光谱和反应混合液的 紫外光谱对比, 发现反应混合液和产物的紫外光谱最大吸收所在波长相同, 因此推测这两个化合物 是反应体系特征性显色的原因。反应过程如图3所示。

从以上实验过程可以看出, 该方法的缺点是只考虑到了反应产物的乙酸乙酯萃取物, 而未考虑 反应体系中的水溶性成分。因此, 随着混合物分离技术及波谱解析技术的进步, Molisch反应机理仍 值得进一步研究验证。

\section{Molisch反应的应用}

布庸置疑, 自从Molisch发现该反应后, 由于其敏锐的反应特点, 很快广泛应用于各种有关糖和 苷的定性研究中。虽然Molisch反应发现时就进行过半定量的实验, 以区别正常尿液和糖尿病尿 ${ }^{[4]}$, 


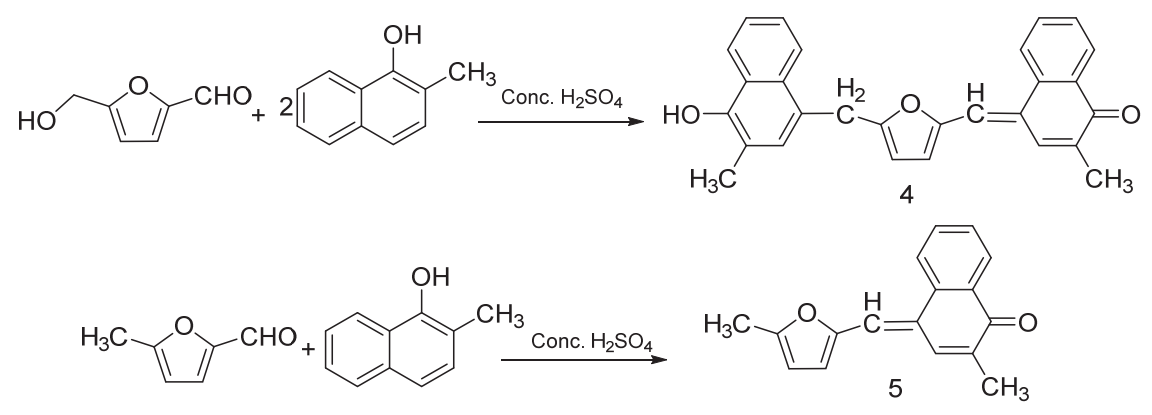

图3 Mutsuko Ohta等的Molisch反应原理

但很长时间以来其定量分析的功能被忽略了。然而, 经过对实验的改进, 确有部分研究者将此反应 用在糖类物质定量分析的尝试上。

Devor A. W. ${ }^{[9,10]}$ 对 Molisch反应进行改进, 以使其更适合于进行糖的比色定量分析。在正常的 Molisch反应中, 也包含了 $\alpha$-䒬酚和浓硫酸的反应, 因此Molisch反应的有色缩合物中除了包含糠醛衍 生物外, 还包含一系列的 $\alpha$-蒜酚磺化物, 这些磺化物的紫外吸收可能干扰比色结果。因此, Devor A.W. 用预先磺化的 $\alpha$-蒜酚代替正常Molisch反应中的 $\alpha$-芸酚, 进行反应后比色测定, 以消除Molisch反应过 程中试剂浓度、温度、加热时间等的干扰, 获得更具重复性的结果。Troelsen J. E. ${ }^{[11]}$ 用类似的方法 测定羊的饲料和粪便中葡萄糖和木糖含量, 确定了饲料的可消化性。

张志华等 ${ }^{[12]}$ 将一定量的葡萄糖溶液和浓硫酸混匀, $70^{\circ} \mathrm{C}$ 水浴反应 $15 \mathrm{~min}$ (产生糠醛衍生物), 放 至室温后加一定量的 $\alpha$-䒬酚, 摇匀放置 $10 \mathrm{~min}$ (产生有色物质), 加无水乙醇稀释至 $10 \mathrm{~mL}$, 稀释至不 同浓度, 在 $570 \mathrm{~nm}$ 处测定吸光度, 绘制工作标准曲线, 表明在葡萄糖浓度在在 $1-50 \times 10^{-6} \mathrm{~g} \cdot \mathrm{mL}^{-1}$ 之 间线性关系良好, 可以用于含量测定, 并测定了木糖醇中还原糖的含量。李勇等 ${ }^{[13]}$ 的发明专利公开 文件中也介绍了一种用类似方法测定木糖醇中还原糖的方法。洪陵成 ${ }^{[14]}$ 的发明专利公开文件中, 介 绍了一种水中糖含量的比色检测方法, 也是用Molish反应后比色的方法进行。杨同香等 ${ }^{[15]}$ 用 Molisch 反应后比色的方法建立了一种测定糖厂汽凝水蔗糖含量的方法。李继民等 ${ }^{[16]}$ 建立了 $\alpha$-萗酚-浓硫酸分 光光度法快速测定不同厂家复印纸水解液中还原糖含量的方法, 为基层民警提供了快速、经济、简 便的纸张物证的鉴定方法。

郑敏燕等 ${ }^{[17]}$ 建立了一种以葡萄糖为标准对照品, 通过 $\alpha$-荎酚-硫酸显色、吸光光度法测定多糖含 量的方法, 用于测定白果多糖的含量。结果显示, 溶液最大吸收波长 $569 \mathrm{~nm}$, 标准葡萄糖溶液在 $0.57-$ $11.5 \mu \mathrm{g} \cdot \mathrm{mL}^{-1}$ 范围内与吸光度之间具有良好的线性关系, 与苯酚-硫酸法相比较, 测量结果基本一致。 张世仙等 ${ }^{[18]}$ 用类似方法测定酒糟多糖的含量, 显示在6-36 $\mu \mathrm{g} \cdot \mathrm{mL}^{-1}$ 范围内吸光度与被测物含量呈良 好的线性关系，重复性好，结果可靠。

\section{5 结语}

Molisch反应发现一百多年来, 在相关领域有关糖和苷类化合物的定性研究中起到不可磨灭的作 用。其反应机理研究由于年代较早, 在很大程度上属于反应过程的推测, 因此值得更进一步厘清, 且 进一步反应机理的研究需同时考虑反应体系中的脂溶性和水溶性反应产物。此外, 以往该反应较少 用在糖或苷类物质的定量分析上, 但根据已有的文献, 该反应在定量分析上极有前景, 因此相关研 究者值得在这方面给予更多关注。

\section{参 考 文 献}

[1] Nature's Editorial Board. Nature 1938, 141, 108.

[2] Gest, H. Photosynth. Res. 1991, 30 (1), 49. 
[3] Herstein, B. J. Am. Chem. Soc. 1910, 32 (6), 779.

[4] Molisch, H. Monatsh. Chem. 1886, 7 (1), 198.

[5] Osborne, T. B.; Harris, I. F. J. Am. Chem. Soc. 1903, 25 (5), 474.

[6] Bredereck, H. Ber. Dtsch. Chem. Ges. 1931, 64B (11), 2856.

[7] Bredereck, H. Ber. Dtsch. Chem. Ges. 1932, $65 B$ (7), 1110.

[8] Ohta, M.; Iwasaki, M.; Kouno, K.; Ueda, Y. Chem. Pharm. Bull. 1985, 33 (7), 2862.

[9] Devor, A. W. J. Am. Chem. Soc. 1950, 72 (5), 2008.

[10] Devor, A. W. J. Agric. Food Chem. 1954, 2 (25), 1290.

[11] Troelsen, J. E. Can. J. Biochem. Physiol. 1961, 39, 1397.

[12] 张志华, 刘育强. 中国食品添加剂, 2004, No. 3, 102.

[13] 李勇, 刘文斌. 木糖醇中还原糖含量的测定方法: 中国, CN201410576923.1 [P]. 2014-10-24.

[14] 洪陵成. 水中糖含量的比色检测方法及装置: 中国, CN201019026061.4 [P]. 2010-02-08.

[15] 杨同香, 陆海勤, 李红, 卢永文. 中国甜菜糖业, 2010, No. 1, 5.

[16] 李继民, 王彦吉, 姚丽娟, 邹宁. 中国刑警学院学报, 2009, No. 2, 58.

[17] 郑敏燕, 耿薇, 魏永生, 张君才. 应用化工, 2010, 39 (3), 447.

[18] 张世仙, 刘炎, 朱涁, 金茜, 曾启华. 食品科学, 2013, 34 (18), 245.

\section{欢迎订阅《物理化学学报》}

2022 年全国报刊杂志征订工作已经开始, 敬请向贵单位推荐订阅 2022 年《物理化学学报》。

《物理化学学报》是由中国科学技术协会主管, 中国化学会和北京大学共同主办, 北京大学化学与分子工程学院学 报编辑部编辑出版的学术刊物。设有 “通讯”、“展望”、“专论”、“综述”、“论文” 等栏目, 报导物理化学学科 及交叉学科的基础研究及应用研究的创新成果。

中国科学院院士唐有祺先生担任名誉主编, 中国科学院院士、北京大学刘忠范教授担任主编; 编委会中有 14 位两 院院士，1 位加拿大科学院院士， 2 位海外学者。2004 年, 获得国内期刊界最高奖一一第三届国家期刊奖百种重点期刊 奖; 2015 年入选国家新闻出版广电总局 “百强报刊”。中国科协精品科技期刊工程项目资助出版。读者普遍认为《物理 化学学报》具有极高的学术价值和收藏价值。WoS 公布的 2020 年影响因子为 2.268 。

《物理化学学报》已被美国《科学引文索引》(SCI)网络版、美国《化学文摘》(CA)、日本《科技文献速报》、俄罗 斯《文献杂志》 $(\mathrm{AJ}) 、$ 、国科协和国家自然基金委主办的《中国学术期刊文摘》、中国科学技术部万方数据网络中心的 《中国科技论文与引文数据库》(CSTPCD)、中国科学院文献情报中心的《中国科学引文数据库》、中国生物学文献数 据中心的《中国生物文摘》等国内外多种著名检索刊物和文献数据库摘引和收录。

《物理化学学报》现为月刊, 大 16 开本, 亚光铜版纸印刷。 2022 年订价 50.00 元/册, 全年订价 600.00 元。国内邮 发代号 82-163, 欢迎国内读者到当地邮局订阅, 国外读者可通过中国国际图书贸易总公司订阅(Code No 1443-MO)。若 通过邮局订阅有困难或错过订阅, 请直接与编辑部联系。欢迎广大科技工作者、科研单位、高等院校、图书馆订阅。

地址: 北京大学化学学院《物理化学学报》编辑部

邮编: 100871

电话: +86-10-62751724

邮箱: whxb@pku.edu.cn

网址: http://www.whxb.pku.edu.cn 